\title{
Longitudinal time-domain optic coherence study of retinal nerve fiber layer in IFN $\beta$-treated and untreated multiple sclerosis patients
}

\author{
REFIK PUL ${ }^{1}$, MEHDI SAADAT ${ }^{1}$, FRANCO MORBIDUCCI ${ }^{1}$, THOMAS SKRIPULETZ ${ }^{1}$, ÜNSAL PUL ${ }^{2}$, \\ DOROTHEE BROCKMANN ${ }^{3}$, KURT-WOLFRAM SÜHS ${ }^{1}$, PHILIPP SCHWENKENBECHER ${ }^{1}$, \\ KAI GÜNTER KAHL ${ }^{4}$, KAWEH PARS ${ }^{1}$, MARTIN STANGEL ${ }^{1}$ and CORINNA TREBST ${ }^{1}$
}

\begin{abstract}
${ }^{1}$ Department of Neurology, Hannover Medical School, 30625 Hannover; ${ }^{2}$ Department of Thoracic and Cardiovascular Surgery, University Hospital Essen, 45147 Essen; Departments of ${ }^{3}$ Ophthalmology and ${ }^{4}$ Psychiatry,
\end{abstract}

Hannover Medical School, 30625 Hannover, Germany

Received June 11, 2015; Accepted February 11, 2016

DOI: $10.3892 / e t m .2016 .3300$

\begin{abstract}
Quantification of the retinal nerve fiber layer (RNFL) by optical coherence tomography (OCT) has been proposed to provide an indirect measure for retinal axonal loss. The aim of the present study was to determine whether interferon beta (IFN $\beta$ ) treatment impedes retinal axonal loss in multiple sclerosis (MS) patients. A total of 48 patients with MS (24 IFN $\beta$-1b-treated and 24 untreated subjects) and 12 healthy controls were enrolled in a prospective longitudinal OCT study. OCT measurements were performed for both eyes of each subject at baseline, and at 3-, 6-, and 12-month follow-up examinations using a time-domain OCT. At each visit, we additionally recorded full-field visual evoked potential (VEP) responses and performed the paced auditory serial addition test (PASAT), in addition to expanded disability status scale (EDSS) scoring. Generalized estimation equation (GEE) was used to account for repeated measurements and paired-data. The model-based approach predicted a monthly reduction in the RNFL thickness by $0.19 \mu \mathrm{m}$ in the eyes of the MS subjects. The reduction was estimated to be $0.17 \mu \mathrm{m}$ in case of IFN $\beta$-treatment and $0.16 \mu \mathrm{m}$ in case of no treatment. Treatment duration and group allocation were not significantly associated with the RNFL thickness. Inclusion of further longitudinal data (EDSS, two and three second PASAT) in each of our models did not result in any significant association. In summary, over a period of one year no significant association between IFN $\beta$-1b treatment and RNFL thinning was identified in patients with MS.
\end{abstract}

Correspondence to: Dr Refik Pul, Department of Neurology, Hannover Medical School, 1 Carl-Neuberg Street, 30625 Hannover, Germany

E-mail: pul.refik@mh-hannover.de

Key words: multiple sclerosis, interferon $\beta-1 \mathrm{~b}$, optical coherence tomography, longitudinal study, retinal nerve fiber layer thickness

\section{Introduction}

Recently increasing attention has been focused on investigating the thickness of the retinal nerve fiber layer (RNFL) in multiple sclerosis (MS) patients. The RNFL contains the proximal portions of the axons that originate from ganglion cell neurons and these axons account for $>80 \%$ of the thickness of this layer (1). Thus, quantification of the RNFL by optical coherence tomography (OCT) has been proposed to provide an indirect appraisal for retinal axonal loss (2-5). As these axons are not myelinated and the visual system is often affected in MS, RNFL measurements in MS patients that suffered from optic neuritis $(\mathrm{ON})$ may offer an effective approach for the study of ON-related neurodegeneration. Indeed, MS patients with a history of ON exhibit reduced RNFL thicknesses compared with healthy control eyes (2-5). It has been reported that the majority of patients sustained a thinning of the RNFL within a period of only 3-6 months following acute ON (4). A significant thinning of the RNFL has been observed in non-affected contralateral eyes in addition to the eyes of MS subjects with no history of ON, suggesting that RNFL damage may occur independently of clinically overt ON $(2,3,5-7)$. Accordingly, post-mortem analyses detected ON lesions in 94-99\% MS subjects, irrespective of any ON history (8-10). However, RNFL reductions were less evident compared with those observed following ON.

It has been demonstrated that following acquired unilateral occipital damage a thinning of the RNFL and optic tract occurs, confirming the existence of retrograde trans-synaptic degeneration of neurons in the human visual pathway (11-13). A significant association between visual cortex damage and RNFL thinning has been shown in MS patients with no ON history, indicating the presence of retrograde trans-synaptic (trans-geniculate) degeneration in the retina (14-16). Conversely, MS subjects who had previously suffered from ON exhibit more intense atrophy in the visual cortex, implicating that the damage cascade may also proceed in anterograde direction $(14,15,17,18)$. Notably, the RNFL thinning in MS patients appears to correlate with global brain atrophy in general, which 
enhances the applicability of using OCT in those subjects, as an association between the RNFL and global measures of disability has been reported by several authors $(3,19-26)$. Human interferon- $\beta$ (IFN $\beta$ ), which is a first-line disease modifying drug for the therapy of relapsing-remitting MS (RRMS), has been shown to have an impact on some of these global measures, including the expanded disability status scale (EDSS) (27-29). However, the proposed neuroprotective effect of IFN $\beta$ is highly controversial $(30,31)$. IFN $\beta$ is speculated to predominantly target the systemic immune response; however, whether it modulates axonal degeneration remains unknown (30,31). Cross-sectional and acute ON OCT studies have not yet provided evidence for any such role $(32,33)$. Thus, the aim of this prospective longitudinal OCT study was to determine whether IFN $\beta$ treatment is able to impede retinal axonal loss.

\section{Materials and methods}

Subjects. A total of 24 IFN $\beta$-1b-treated and 24 subjects with untreated RRMS according to revised McDonald criteria (2005) were enrolled (34). The untreated cohort consisted of subjects who refused MS disease-modifying therapy $(n=17)$ or did not receive it for $>5$ years $(n=7)$. The following data were obtained from each MS patient at the screening visit: Age, sex, co-morbidities, co-medication, date of MS symptom onset, disease duration, prior episodes of $\mathrm{ON}$, prior MS medication, total number of relapses, last relapse, and, for the IFN $\beta-1 b$ group, start date of IFN $\beta$-1b therapy. Disease duration was defined as the time period between the first recognized symptoms of MS and study inclusion. Subjects were aimed to broadly match for age, gender and refraction ( \pm 2 diopters). None of the enrolled MS subjects sustained an acute relapse and/or received systemic steroid treatment within 30 days prior to study entry. The untreated MS patients had been without immunomodulatory treatment for at least one year prior to study entry. None of the subjects were previously treated with teriflunomide, mitoxantrone or cyclophosphamide. In addition, none of the subjects suffered from any ophthalmological or neurological disorder other than MS. The control group consisted of 12 healthy volunteers with no history of ocular or neurological disease and with a visual acuity of $\geq 1.0$. The study was approved by the Institutional Review Board of the Hannover Medical School (Hannover, Germany). All participants signed an informed consent form detailing the purpose of this study, the tests included in the exploratory protocol, and the permission to stop participating in the study at any time.

Clinical assessment. The present study was a prospective longitudinal study over a period of one year. Subjects were evaluated at baseline and at 3,6 and 12 months. All subjects underwent a complete ophthalmic examination that included assessment of best-corrected visual acuity, ocular motility, pupillary reflexes, biomicroscopy of the anterior segment using a slit lamp, papillary morphology with fundoscopic exam, and visual field examination of each eye at baseline visit. At each visit, visual acuity tests were performed using Landolt rings and RNFL thicknesses were quantified by OCT. All examinations were conducted by a team of ophthalmologists, optometrists and orthoptists.
In addition, the EDSS score and the two/three second paced auditory serial addition test (PASAT) were assessed at each visit. The EDSS score was ascertained by trained neurologists, while the PASAT was performed by a trained study nurse. At each visit, full-field VEP were recorded monocularly in a dark room after occlusion of the other eye and elicited by $1.3 \mathrm{~Hz}$ pattern reversal of a $50 \%$ contrast black-and-white checkerboard at a viewing distance of $1 \mathrm{~m}$ (Viking Nicolet Quest; Natus Medical Incorporated, Soltau, Germany). Silver chloride-plated disk electrodes (GVB-geliMED, Bad Segeberg, Germany) were placed on the scalp at the occipital (active electrode) and frontal (reference electrode) areas. The latency and amplitude (peak-to-peak amplitude of N75-P100) of the positive fundamental component (P100) were analyzed.

RNFL imaging. OCT was performed with a time-domain (TD) OCT (Stratus OCT Model 3000; Carl Zeiss Meditec AG, Jena, Germany). RNFL images were acquired by taking three circular 3.4-mm scans, centered on the optic disc, the mean of which was used to express RNFL thickness (Fast RNFL thickness protocol). The ophthalmology team which participated in this study complied with the majority of the points listed in the OSCAR-IB criteria, such as retinal pathology, obvious problems, centration of scans, control of algorithm failure, illumination and beam placement. However, a signal strength of $\geq 8$ was considered acceptable in this study, whereas signal strength of $>15$ is required to meet the OSCAR-IB criteria $(35,36)$.

Statistical analysis. Analyses were conducted using generalized estimating equation (GEE) models with an exchangeable working correlation structure to account for correlation between the two eyes from a single participant $(37,38)$. As it is well known that the GEE estimator of the variance-covariance matrix of the parameter estimates leads to inflated Type I error rates, the robust covariance matrix was gauged via an iterative jackknife resampling method (39). The free software programming language $\mathrm{R}$ version 3.1 .2 (https://cran.r-project.org.) with the package geepack was used to perform all GEE analyses (40). For pairwise comparison of the predicted marginal means, the R Package doBy was used. One-way analysis of variance, Kruskal-Wallis test (comparison of three independent groups) and the two-tailed Mann-Whitney U test (comparison of two independent groups) were used for additional statistical analysis according to data distribution as tested by the Shapiro-Wilk test. For the latter, analyses were performed using GraphPad Prism, version 5.02 (GraphPad Software, Inc., La Jolla, CA, USA).

OCT scans were performed by more than one ophthalmologist. Repeated RNFL data of healthy controls were used to perform Bland-Altman analysis (data not shown). Prior to Bland-Atman analysis, normal distribution of data were confirmed by the Shapiro-Wilk test and relative and proportional bias were excluded by one-sample t-test and linear regression, respectively (data not shown). The Bland-Altman method is used to compare two measurement techniques but it can also be applied to two measurements obtained with the same technique (41). The resulting Bland-Altman plots showed that $95 \%$ of the difference scores lie between the limits of agreement as requested for this method (data not shown). 
Table I. Demographic characteristics.

\begin{tabular}{lcccccr}
\hline & & & & \multicolumn{2}{c}{ Age (years) } \\
\cline { 5 - 7 } Group & $\mathrm{n}$ & Female & Male & Mean & Minimum & Maximum \\
\hline Healthy controls & 12 & 9 & 3 & $41.67 \pm 9.82$ & 26 & 61 \\
Untreated MS & 24 & 20 & 4 & $46.46 \pm 11.09$ & 29 & 69 \\
IFN $\beta$-treated MS & 24 & 20 & 4 & $45.63 \pm 10.54$ & 28 & 63 \\
\hline
\end{tabular}

MS, multiple sclerosis; IFN $\beta$, interferon- $\beta$.

Table II. Clinical characteristics.

\begin{tabular}{|c|c|c|}
\hline Parameter & Untreated MS & IFN $\beta$-treated MS \\
\hline \multicolumn{3}{|l|}{ Duration of IFN $\beta$ treatment, years } \\
\hline Mean & N/A & $2.6 \pm 3.1$ \\
\hline Medium & $\mathrm{N} / \mathrm{A}$ & 1 \\
\hline \multicolumn{3}{|l|}{ EDSS } \\
\hline Mean & $2.2 \pm 0.8$ & $2.3 \pm 1.1$ \\
\hline Medium & 2 & 2 \\
\hline \multicolumn{3}{|l|}{ Disease duration, years } \\
\hline Mean & $13.6 \pm 9.8$ & $8.3 \pm 6.0^{*}$ \\
\hline Median & 10 & 8 \\
\hline \multicolumn{3}{|l|}{ Relapses prior to study, $\mathrm{n}$} \\
\hline Mean & $5.4 \pm 4.8$ & $4.5 \pm 3.6$ \\
\hline Medium & 5 & 3 \\
\hline \multicolumn{3}{|c|}{ Time from last relapse to study inclusion, years } \\
\hline Mean & $1.8 \pm 2.3$ & $1.6 \pm 2.1$ \\
\hline Medium & 1 & 1 \\
\hline Relapses during study, $\mathrm{n}$ & 6 & 7 \\
\hline Total subjects with MS relapse, $\mathrm{n}$ & 6 & 6 \\
\hline
\end{tabular}

One-way analysis of variance was used for statistical analyses, followed by Kruskal-Willis test, when comparing three independent groups and two-tailed Mann-Whitney $\mathrm{U}$ test, when comparing two independent groups, according to the normality of the data. " $\mathrm{P}<0.01$ vs. the untreated MS group. IFN $\beta$, interferon- $\beta$; MS, multiple sclerosis; EDSS, expanded disability status scale.

The limits of agreement revealed test-retest variability. Thus, intraclass correlation coefficients (ICC) with a two-way mixed-effects model for measures of absolute agreement as setting were assessed. The obtained inter-rater ICC ranged between 0.89 and 0.97 , indicating the 'sufficient' consistency of the measurements. To estimate noise by test-retest variation, single courses of repeated OCT values are displayed in Fig. 1.

\section{Results}

Demographics and clinical characteristics. The demographic details are summarized in Table I. The study analyzed 96 eyes from 48 patients with MS and 24 eyes from 12 healthy controls. The majority of the participants were women: $80 \%$ in among the treated and untreated MS groups and $66.7 \%$ in the control group. The mean and median age of subjects was comparable between the two MS groups. Although the EDSS score ranged between 1 and 4 in the untreated and 0 and 6 in the IFN $\beta$-treated MS group, the mean and median EDSS was almost identical in both MS groups. Subjects in the untreated group had more relapses prior to study entry and significantly longer disease duration (Mann-Whitney $\mathrm{U}$ test, $\mathrm{P}=0.0078$, $\mathrm{U}=790.0$ ). The mean time since last relapse until study inclusion did not differ significantly between both MS groups. During the study period, six subjects suffered relapses in each MS group. Despite IFN $\beta$ treatment, relapses occurred slightly more frequently in the IFN $\beta$-treated group (Tables I and II). The patient history revealed that 15 of the untreated and 18 of the IFN $\beta$-treated MS patients sustained ON (Table III). During the study period, only two subjects suffered from ON and both of them belonged to the group of untreated MS patients.

RNFL thickness is decreased only in the eyes of MS subjects with prolonged VEP latencies. As further analyses were conducted 


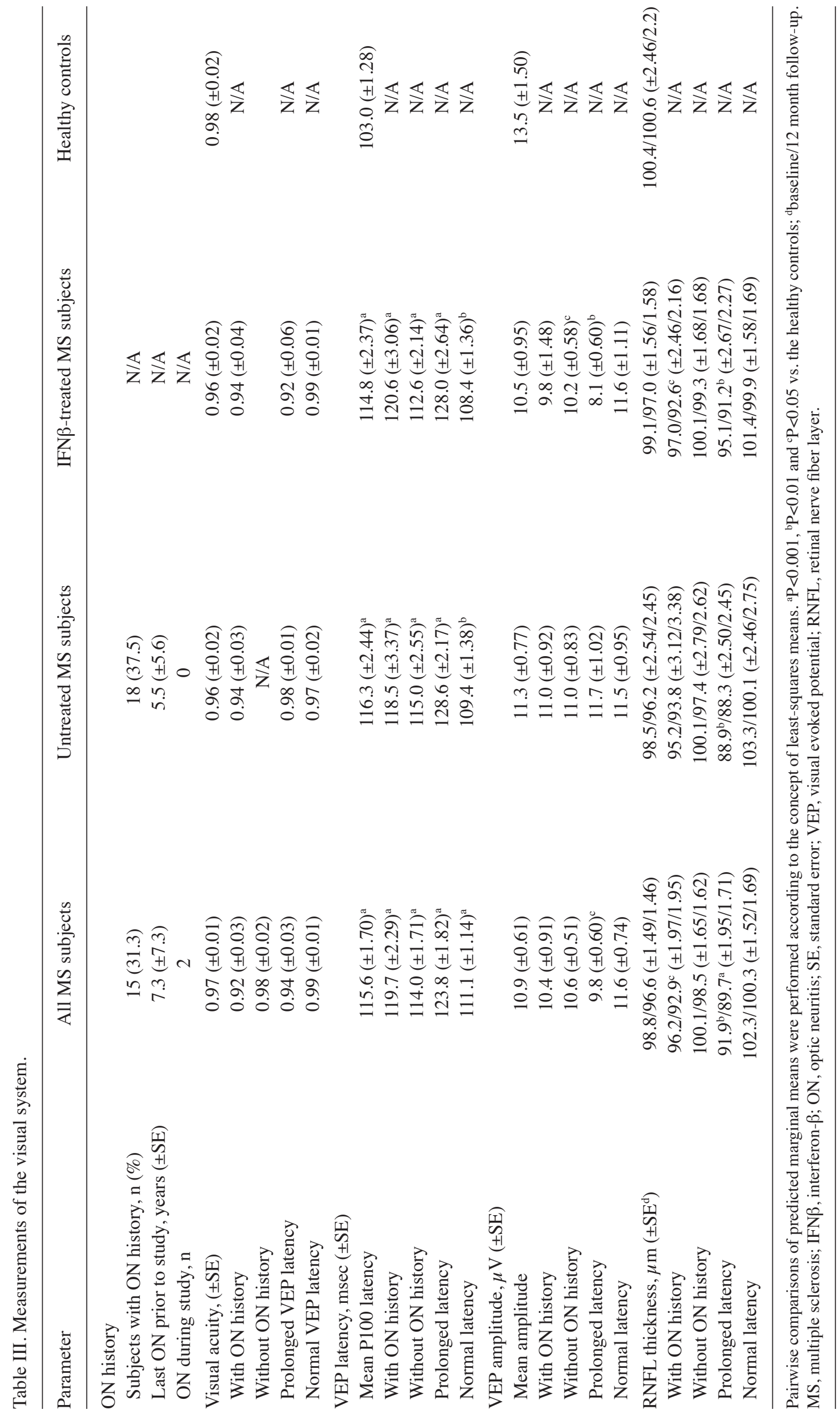


Table IV. Generalized analyses of different covariates and their association with the RNFL thickness in the healthy controls and entire MS cohort.

$95 \%$ Wald CI

\begin{tabular}{|c|c|c|c|c|c|c|}
\hline Parameter & $\mathrm{B}$ & SE & Lower & Upper & Wald $\chi^{2}$ & P-value \\
\hline \multicolumn{7}{|l|}{ Healthy control subjects $(n=24)^{a}$} \\
\hline Intercept value & 111.21 & 4.03 & 14.14 & 29.95 & 230.70 & $<0.001$ \\
\hline Time per month & 0.02 & 0.05 & -0.08 & 0.11 & 0.10 & 0.754 \\
\hline Age per year & -0.27 & 0.16 & -0.58 & 0.05 & 2.81 & 0.090 \\
\hline \multicolumn{7}{|l|}{ All MS subjects $(\mathrm{n}=96)^{\mathrm{b}}$} \\
\hline Intercept value & 94.93 & 6.84 & 81.53 & 108.34 & 192.61 & $<0.001$ \\
\hline Time per month & -0.19 & 0.04 & -0.28 & -0.11 & 21.21 & $<0.001$ \\
\hline Age per year & 0.18 & 0.17 & -0.15 & 0.52 & 1.13 & 0.287 \\
\hline Disease duration per year & -0.61 & 0.17 & -0.95 & -0.27 & 14.25 & $<0.001$ \\
\hline Total relapses per relapse & 0.48 & 0.28 & -0.07 & 1.03 & 2.89 & 0.089 \\
\hline \multicolumn{7}{|c|}{ VEP latency and amplitude of all subjects $(n=120)^{c}$} \\
\hline Intercept value & 118.95 & 8.13 & 103.01 & 134.88 & 214.00 & $<0.001$ \\
\hline VEP latency per msec & -0.21 & 0.07 & -0.34 & -0.07 & 9.22 & 0.002 \\
\hline VEP amplitude per $\mu \mathrm{V}$ & 0.29 & 0.10 & -0.09 & 0.49 & 7.73 & 0.005 \\
\hline \multicolumn{7}{|l|}{ ON history of MS subjects $(n=96)^{d}$} \\
\hline Intercept value & 100.63 & 2.52 & 95.69 & 105.58 & $1,590.71$ & $<0.001$ \\
\hline Group with no ON history & 2.98 & 1.49 & 0.06 & 5.90 & 4.01 & 0.045 \\
\hline Time per month (no ON history) & -0.18 & 0.05 & -0.28 & -0.08 & 12.76 & $<0.001$ \\
\hline Time per month (ON history) & -0.23 & 0.07 & -0.37 & -0.08 & 9.59 & 0.002 \\
\hline ON vs. ON history & N/A & 1.49 & 0.05 & 5.88 & 3.96 & 0.046 \\
\hline \multicolumn{7}{|c|}{ Prolonged VEP latency of MS subjects $(n=96)^{e}$} \\
\hline Intercept value & 96.16 & 3.02 & 90.25 & 102.08 & $1,014.82$ & $<0.001$ \\
\hline Normal group & 7.99 & 2.00 & 4.07 & 11.91 & 15.94 & $<0.001$ \\
\hline Time per month (normal) & -0.20 & 0.05 & -0.31 & -0.10 & 15.00 & $<0.001$ \\
\hline Time per month (prolonged) & -0.18 & 0.06 & -0.30 & -0.06 & 8.67 & 0.003 \\
\hline Prolonged vs. normal & $\mathrm{N} / \mathrm{A}$ & 1.99 & 3.96 & 11.75 & 15.65 & $<0.001$ \\
\hline
\end{tabular}

Pairwise comparisons of predicted marginal means were performed according to the concept of least-squares means. Significant P-values are indicated in bold. 'Dependent variables: Retinal nerve fiber layer (RNFL) thickness; model, (intercept); time; age; 'bependent variables: RNFL thickness; model: (intercept); time; age; disease duration; total number of relapses; 'Dependent variables: RNFL thickness; model: (intercept);

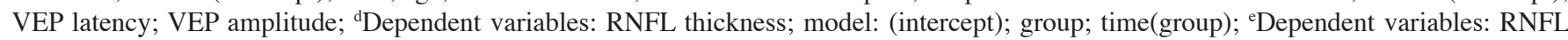
thickness; model: (intercept); group; time(group). CI, confidence interval; B, regression coefficient; SE, standard error; MS, multiple sclerosis; VEP, visual evoked potential; ON, optic neuritis.

on paired data, estimated marginal means were assessed using the GEE approach and for pairwise comparisons (Table III). In addition to IFN $\beta$ treatment or non-treatment, untreated MS subjects were additionally grouped to those with/without ON and those with/without prolonged VEP latency. The subgroup prolonged VEP latency was selected as the ON history of each MS subject partially coincided with prolonged VEP latencies. In total, 18 eyes of the untreated ( 6 with prior ON history) and 14 eyes of the IFN $\beta$-treated MS subjects (10 with prior ON history) exhibited prolonged VEP latencies at baseline.

Considering estimated marginal means, the visual acuity did not significantly differ between all groups (Table III). As expected, MS subjects exhibited longer P100 latencies of the VEP compared to healthy controls (Table III). However, in all subgroups there was no statistical difference in VEP latency between IFN $\beta$-treated and untreated MS subjects (Table III). Next, the amplitudes of the P100 wave were compared between the groups. The VEP amplitudes tended to be lower in the eyes of IFN $\beta$-treated MS subjects, and were significantly lower in cases with ON history or prolonged VEP latency compared to VEP amplitudes obtained from healthy control eyes (Table III). A statistical difference in VEP amplitudes was detected between MS subjects with prolonged and normal VEP latency $(\mathrm{P}<0.001)$. However, there was no statistically significant difference in VEP amplitudes between IFN $\beta$-treated and untreated MS subjects in all subgroups (Table III).

Baseline and 12-month follow-up data for RNFL thickness are shown in Table III. Without classification to any subgroup, there was no difference in the estimated marginal means of the RNFL thicknesses between healthy control eyes and the eyes 
Right eye
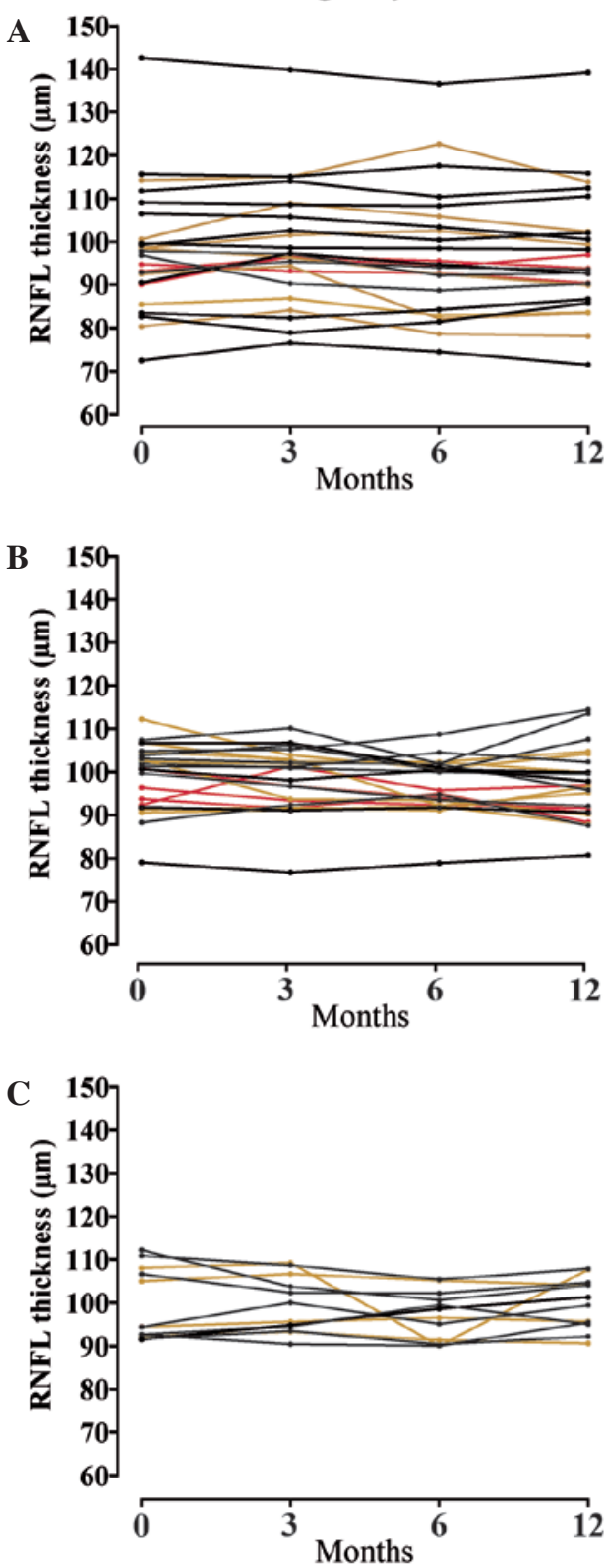

Left eye
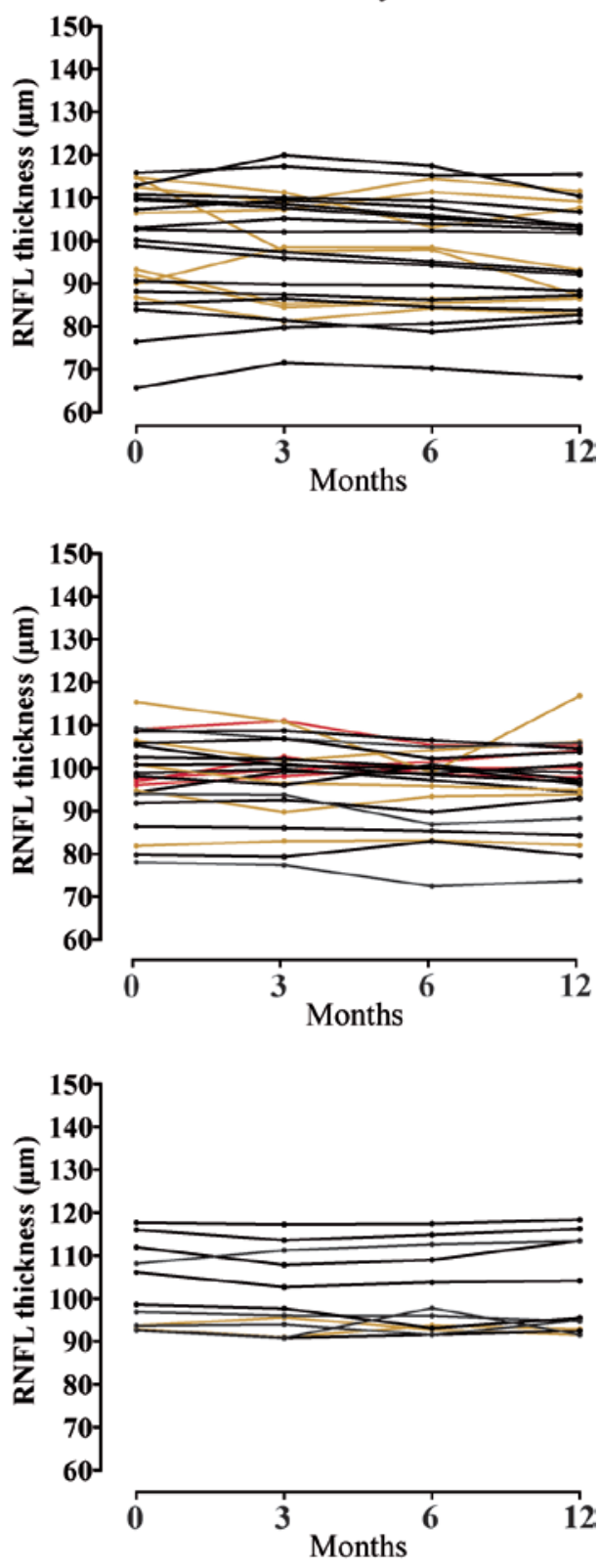

Figure 1. Single courses of repeated RNFL values in (A) untreated MS subjects, (B) interferon- $\beta$-treated MS subjects and (C) healthy controls. To ensure a better overview, RNFL values are displayed according to the right or left eye, respectively. Colors are used to delineate lines that would otherwise be indiscernible. RNFL, retinal nerve fiber layer; MS, multiple sclerosis.

of MS subjects at baseline ( $\mathrm{P}=0.848)$. Similarly, no differences in RNFL thickness were observed between healthy control eyes and the eyes of MS subjects with or without ON history (GEE, $\mathrm{P}=0.375$ and $\mathrm{P}=0.992$, respectively). Notably, eyes of MS subjects with prolonged VEP latencies, in particular those of untreated MS subjects, exhibited significantly lower RNFL thicknesses compared with healthy subjects (GEE, $\mathrm{P}=0.007$ and $\mathrm{P}=0.001$, respectively). At the 12-month follow-up OCT examination, a significantly lower RNFL thickness was detected in the eyes of MS subjects with ON history compared with control eyes. Again, there was no statistical difference in the RNFL thicknesses between IFN $\beta$-treated and untreated MS subjects.

IFN $\beta$-treatment does not have an impact on RNFL thinning. GEE model-based approaches were used for investigation of covariates. The design of each model and results are summarized in Tables IV and V. The covariates age and total number of (non-visual) relapses did not contribute significantly to our GEE models (Table IV). MS disease duration was strongly associated with the RNFL thickness and each year was associated with an annual reduction in the RNFL thickness by $0.61 \mu \mathrm{m}$ (Table IV). The regression coefficient B of the covariate time predicted a monthly decrease in the RNFL thickness by $0.19 \mu \mathrm{m}$ in eyes of MS subjects, while this covariate was not predictive in healthy control eyes (Table IV). A history of ON was associated with lower RNFL thicknesses, while having no ON history was associated with higher RNFL thicknesses (Table IV). Time was associated with a monthly reduction in the RNFL thickness by $0.23 \mu \mathrm{m}$ in cases with ON history and $0.18 \mu \mathrm{m}$ in MS subjects without ON history (Table IV). 
Table V. Generalized analyses of treatment covariates and their association with the RNFL thickness; allocation to treatment or no-treatment.

95\% Wald CI

Parameter

B

SE

Lower Upper

Wald $\chi^{2} \quad$ P-value

All MS subjects $(n=96)$

Intercept value

99.51

Group with no treatment

Time per month (no treatment)

Time per month (treatment)

Treatment duration

No treatment vs. IFN $\beta$ treatment

MS subjects with ON history $(n=33)$

Intercept value

Group with no treatment

Time per month (no treatment)

Time per month (treatment)

Treatment duration

No treatment vs. IFN $\beta$ treatment

MS subjects without ON history $(n=63)$

Intercept

Group with no treatment

Time per month (no treatment)

Time per month (treatment)

Treatment duration

No treatment vs. IFN $\beta$ treatment

MS subjects with prolonged VEP latencies $(n=32)$

Intercept value

Group with no treatment

Time per month (no treatment)

Time per month (treatment)

Treatment duration

No treatment vs. IFN $\beta$ treatment

MS subjects with normal VEP latencies $(n=64)$

Intercept value

Group with no treatment

Time per month (no treatment)

Time per month (treatment)

Treatment duration

No treatment vs. IFN $\beta$ treatment

$\begin{array}{rrrrcr}99.51 & 2.78 & 94.05 & 104.96 & 1,278.52 & <\mathbf{0 . 0 0 1} \\ 5.39 & 4.09 & -2.63 & 13.41 & 1.74 & 0.188 \\ -0.16 & 0.06 & -0.32 & -0.09 & 11.78 & \mathbf{0 . 0 0 1} \\ -0.17 & 0.06 & -0.30 & -0.07 & 10.51 & \mathbf{0 . 0 0 1} \\ 0.77 & 0.40 & -0.08 & 1.55 & 3.77 & 0.052 \\ \text { N/A } & 4.10 & -2.75 & 13.31 & 1.66 & 0.197\end{array}$

$\begin{array}{rrrrrr}123.18 & 14.04 & 95.66 & 150.69 & 76.99 & <\mathbf{0 . 0 0 1} \\ 2.04 & 5.43 & -8.60 & 12.67 & 0.14 & 0.707 \\ -0.02 & 0.11 & -0.24 & 0.20 & 0.04 & 0.852 \\ -0.42 & 0.12 & -0.66 & -0.18 & 11.82 & \mathbf{0 . 0 0 1} \\ 0.78 & 0.58 & -0.35 & 1.92 & 1.84 & 0.175 \\ \text { N/A } & 5.48 & -6.65 & 14.83 & 0.56 & 0.456 \\ & & & & & \\ 98.90 & 6.86 & 85.45 & 112.35 & 207.68 & <\mathbf{0 . 0 0 1} \\ 8.02 & 4.13 & -0.87 & 16.12 & 3.76 & 0.053 \\ -0.023 & 0.11 & -0.45 & -0.01 & 4.26 & \mathbf{0 . 0 3 9} \\ -0.30 & 0.08 & -0.20 & 0.14 & 0.013 & 0.723 \\ 0.95 & 0.44 & 0.09 & 1.81 & 4.64 & \mathbf{0 . 0 3 1} \\ \text { N/A } & 4.08 & -0.92 & 15.09 & 3.00 & \mathbf{0 . 0 8 3}\end{array}$

$\begin{array}{rrrrrr}114.32 & 15.51 & 83.92 & 144.72 & 54.33 & <\mathbf{0 . 0 0 1} \\ -4.35 & 6.37 & -16.83 & 8.14 & 0.47 & 0.495 \\ -0.01 & 0.11 & -0.20 & 0.22 & 0.02 & 0.903 \\ -0.42 & 0.12 & -0.66 & -0.18 & 11.38 & \mathbf{0 . 0 0 1} \\ 0.66 & 0.60 & -0.52 & 1.84 & 1.20 & 0.273 \\ \text { N/A } & 6.06 & -14.28 & 9.48 & 0.16 & 0.692 \\ & & & & & \\ 103.27 & 2.74 & 97.91 & 108.64 & 1,425.29 & <\mathbf{0 . 0 0 1} \\ 10.15 & 3.58 & 3.13 & 17.17 & 8.03 & \mathbf{0 . 0 0 5} \\ -0.30 & 0.08 & -0.45 & -0.15 & 14.71 & \mathbf{0 . 0 0 0} \\ -0.12 & 0.06 & -0.24 & 0.01 & 3.29 & 0.070 \\ 0.97 & 0.52 & -0.06 & 2.00 & 3.43 & 0.064 \\ \text { N/A } & 3.29 & 1.89 & 14.79 & 6.42 & \mathbf{0 . 0 1 1}\end{array}$

Pairwise comparisons of predicted marginal means were performed according to the concept of least-squares means. Significant P-values are indicated in bold. CI, confidence interval; B, regression coefficient; SE, standard error; IFN $\beta$, interferon- $\beta$; MS, multiple sclerosis; ON, optic neuritis; RNFL, retinal nerve fiber layer; VEP, visual evoked potential.

Prolonged VEP latency was associated with a reduction in the RNFL thickness by $7.99 \mu \mathrm{m}$. The covariate time, however, did not show an appreciable difference in the regression coefficients between subjects with and without prolonged VEP latency (Table IV). Comparison of the marginal means confirmed significantly higher RNLF thickness values in MS subjects without ON history $(98.73 \pm 1.50 \mu \mathrm{m})$ or normal VEP latency $(100.53 \pm 1.32 \mu \mathrm{m})$ compared with those with ON history
$(95.75 \pm 1.71 \mu \mathrm{m})$ or prolonged P100 latency $(92.55 \pm 1.97 \mu \mathrm{m})$ of the VEP, respectively (Table IV).

In a second set of GEE analyses, we analyzed the association between treatment and the RNFL thickness when controlling for group (IFN $\beta$ treatment or no treatment) and treatment duration. Time, disease duration, VEP latency and amplitude were also entered in each model and the results are shown in Table V. Considering all MS subjects, no apparent 
difference was identified in the predicted monthly decrease of the RNFL thickness. The decrease was estimated to be $0.17 \mu \mathrm{m}$ in case of IFN $\beta$-treatment and $0.16 \mu \mathrm{m}$ in case of no treatment. Treatment duration and group allocation were not significantly associated with the RNFL thickness, underlining that treatment in general was not predictive for the RNFL thickness (Table V). In subgroup analyses, there were discrepant results. Accordingly, significant negative associations were found for the covariate time in MS subjects without ON and normal P100 latency of the VEP in case of no treatment, while in case of treatment such associations were found for MS subjects with ON and prolonged P100 latency of the VEP (Table V). These findings may allude to heterogeneity among our MS cohort. Notably, treatment duration showed a significant positive association with the RNFL thickness, but only in MS subjects without ON history. Furthermore, in this subgroup group allocation did not reveal an association between RNFL thickness and treatment (Table V). In the subgroup of MS subjects with normal VEP latency, comparison of the marginal means confirmed significantly higher RNLF thickness values in untreated MS subjects $(105.97 \pm 2.33 \mu \mathrm{m})$ compared with IFN $\beta$-treated subjects $(97.63 \pm 1.72 \mu \mathrm{m})$ (Table V).

Inclusion of further longitudinal data (EDSS, two and three second PASAT) in each of the above mentioned GEE models did not result in any significant associations (data not shown). Notably, since analysis of quadrant data using Stratus OCT is highly prone to artefacts, particularly in longitudinal measurements, and probably will to a certain extent reflect eye position changes in subjects rather than actual measurement differences, quadrant data were not analyzed.

\section{Discussion}

RNFL thickness is considered to be a relevant parameter to infer axonal loss in patients with MS and/or ON. Although stipulated in numerous studies, the number of longitudinal studies and particularly those which investigated possible drug effects on the RNFL in MS is limited $(6,7,21)$. The study conducted by Talman et al (42), which included 593 eyes, was among the first longitudinal OCT studies in MS patients. Using a TD OCT, they observed a consistent decline in RNFL thickness in the eyes of MS patients, even in those without history of ON. Although in that study $87 \%$ of subjects were receiving disease modifying therapies, treatments were not specified (42). In another longitudinal study (43), which included a total of 155 eyes, TD OCT was performed at two time points, with an interval of one year. Treatment groups were well classified (IFN $\beta-1 a$, IFN $\beta-1 b$, and glatiramer acetate); however, the number of study participants per group was not stated. After a follow-up of one year, no differences in RNFL loss were identified in treated compared with the untreated MS subjects. With the exception of these two studies, there are no other longitudinal OCT studies addressing the question whether classical first-line treatment of MS (IFN $\beta$ and glatiramer acetate) may impede RNFL thinning.

In the present study, GEE models were used to identify significant associations between IFN $\beta$ treatment and the peripapillary RNFL thicknesses obtained from four defined visits over a period of one year. According to the present model-based approach, the RNFL loss in the total MS cohort was estimated to be $0.16 \mu \mathrm{m}$ per month of disease, which is consistent with the RNFL loss observed in a previous study by Talman et al (42). Garcia-Martin et al (43), the authors of the aforementioned second longitudinal study, have reported a reduction in RNFL thickness of $3.48 \mu \mathrm{m}$ per year. However, this discrepancy may be ascribable to their different statistical approach, as the study by Talman et al (42) and the present study employed a GEE method adjusted for within-patient and inter-eye correlations. In other longitudinal studies following different objectives, RNFL thinning rates ranging between 0.3 and $4.6 \mu \mathrm{m}$ per year in eyes of MS subjects have been reported (14,44-47). Besides differences in statistical approaches, variations in the rate of RNFL thinning may be explained by the composition of cohorts, disease course and disease duration. The distinction between benign (EDSS, $\leq 3$; disease duration, $\geq 15$ years) and classical MS appears to play no role in the observed differences (44). In the present study, the monthly reduction in untreated MS subjects was predicted to be 0.16 and $0.17 \mu \mathrm{m}$ in IFN $\beta$-treated MS subjects. Treatment duration and group allocation as independent and time-invariant covariates did not reveal a significant association with RNFL thickness. Subgroup analyses, however, provided disparate results, which was construed as heterogeneity among the present MS cohort. However, none of these analyses indicated that IFN $\beta$ treatment may have an impact on the rate of RNFL loss.

According to Henderson et al (47), RNFL thinning is most probably not a linear process. They speculate that there is more rapid RNFL loss in earlier RRMS when subclinical inflammatory demyelination is common and may involve the optic nerve, suggesting that the dynamics of RNFL thinning may alter during the MS course (47). The lack of knowledge regarding the time course of RNFL loss generally complicates the interpretation of longitudinal OCT studies irrespective of whether time-domain or newer generation of this device is used, such as spectral domain OCT. It remains unclear whether this may have played a role in the longitudinal OCT study of Serbecic et al (high resolution spectral domain OCT, 27 subjects with RRMS and 10 subjects with secondary progressive MS; observation period of $\sim$ two years) and Henderson et al (TD OCT, 18 subjects with primary progressive and 16 with secondary progressive MS, observation period of $\sim 18$ months) $(47,48)$. Measuring the RNFL loss following acute $\mathrm{ON}$ would enable the study of a well-defined time frame of pathology. Accordingly, Suehs et al examined changes in RNFL thickness in subjects with clinically isolated syndrome manifested as acute ON. However, at this rather early point of disease, they did not detect any effect of IFN $\beta$ on RNFL thinning (32).

In a number of previous studies, as summarized in a meta-analysis by Petzold et al (49), a clear RNFL reduction compared to healthy controls has been reported. The present sample size is most probably too small to detect such a difference and, in addition, the majority of these previous studies enrolled MS subjects with visual impairments, which may explain lower RNFL thicknesses in their cohorts. The vast majority of MS subjects that were enrolled in the present study had a stable visual acuity of 1.0, as assessed using Landolt ring tests. This may explain higher RNFL thicknesses and the lack of association between visual acuity and the RNFL thickness in the present MS cohort. In general, visual acuity 
testing appears to be a relatively insensitive measure of optic nerve dysfunction in MS. Low-contrast visual acuity, visual field testing, color vision, and stereopsis have been shown to more effectively capture visual impairment among MS subjects $(3,4,50,51)$. In the present study, prolongation of the latency remained as a functional surrogate marker, which elicited visual pathway dysfunction. Furthermore, the eyes of MS subjects with prolonged latencies exhibited lower RNFL thicknesses compared with healthy control eyes and were associated with a strong RNFL reduction of $7.99 \mu \mathrm{m}$, indicating that demyelination is involved in promoting axonal loss. In addition, both VEP parameters (i.e., amplitude and P100 latency) revealed a significant association with the RNFL thickness, corroborating the results of previous studies $(2,52)$.

As expected, MS subjects with ON history exhibited the highest rate in RNFL thinning, however, the predicted reduction of $0.23 \mu \mathrm{m}$ per month was slightly higher than in eyes without ON history. Such a slight difference between eyes with and without $\mathrm{ON}$ has been previously reported by Talman et al (42). In addition, Talman et al observed that healthy control eyes also experience RNFL thinning, which was estimated to be $0.49 \mu \mathrm{m}$ over a 3 -year period (28). The shorter duration of the present study, the relatively small cohort size and the fact that the structural changes in RNFL thickness may have been below the detection limit of the TD OCT, may have played a role in not detecting RNFL thinning in the control cohort.

Toledo et al (20) observed a marked correlation between RNFL thickness and a visually dependent cognitive test (symbol digit modality test), indicating that RNFL loss may reflect similar pathological changes taking place in the brain. Among other cognitive tests, Toledo et al identified a significant correlation between the average RNFL thickness and the three second PASAT. However, the authors did not focus on this result as they did not detect a correlation with the thickness in the temporal quadrant of the RNFL. In the present study, two and three second PASAT was selected as a non-visual test, and no association was detected with the RNFL thickness. Notably, visual dysfunction has been shown to potentially impair test performance in visually dependent cognitive tests, suggesting that those tests are more likely to show correlation with RNFL parameters than non-visual tests $(53,54)$. It seems conceivable that this association may also hold true in the other direction, i.e. decline of visual test performance by cognitive impairment (55). The EDSS inadequately captures dysfunction within the visual system, which may explain conflicting results of a previous study (49). No association between the EDSS and RNFL thickness was detected in the present study.

Little is known about the effects of IFN $\beta$ on axonal preservation. Increased neuronal survival in vitro and increased nerve growth factor concentrations in glial and brain cell cultures that were exposed to IFN $\beta$ have been reported (56-58). Recently, IFN $\beta$ has been shown to regulate genes involved in neuronal preservation, such as the nuclear factor erythroid 2-related factor 2, and genes involved in energy metabolism, such as the inhibition of IкB kinase (IKK) and IKK-related kinases (59). A previous clinical trial revealed an inconsistent impact of IFN $\beta$ on general brain atrophy (60). A reanalysis of the BEYOND study, which was a large, phase III, clinical trial comparing IFN $\beta-1 b$ treatment in two different doses $(250$ and $500 \mu \mathrm{g})$ and glatiramer acetate, showed that IFN $\beta-1 b$ therapy was associated with a reduction in magnetic resonance imaging-detected permanent black hole formation and evolution, suggesting a possible neuroprotective effect (61). As black holes are multiple sclerosis plaques detected in the chronic stage during axonal destruction, they are indicative of neurodegeneration in MS (62). With respect to the RNFL, the present data do not indicate that IFN $\beta$ is able to deter neurodegeneration, although a neuroprotective effect by IFN $\beta$ therapy may be conceivable by preventing optical relapses. MS subjects without $\mathrm{ON}$ appear to be an ideal cohort for investigating the neuroprotective properties of IFN $\beta$ on RNFL. However, it remains unclear whether axonal loss in eyes without $\mathrm{ON}$ history is a result of several episodes of subclinical $\mathrm{ON}$ or may be attributed to an unknown mechanism of neurodegeneration. Reliable criteria to detect subclinical ON have not yet been established.

In conclusion, over a period of one year no significant association between IFN $\beta$ - $1 b$ treatment and RNFL thinning was observed in MS subjects. The inherent limitation of this study is the use of TD OCT, which has higher test-retest variability compared with more recent spectral domain technology. Crucially, measurement values from these different techniques are not interchangeable (63). Longitudinal studies that include a longer period are required not only to examine treatment effects but also the dynamics of RNFL thinning.

\section{Acknowledgements}

This study was financially supported by Bayer HealthCare (Berlin, Deutschland) and is part of Mehdi Sadat's MD thesis. For electrophysiological measurements, the authors thank Ms. Mandy Wenzel, Ms. Madeleine Rambow, Ms. Patrizia Gerstenberger and Ms. Claudia Wilmsmann (Department of Neurology, Hannover Medical School). The authors thank Dr Jelena Skuljec for reviewing this paper.

\section{References}

1. Wirtschafter JD: Optic nerve axons and acquired alterations in the appearance of the optic disc. Trans Am Ophthalmol Soc 81: 1034-1091, 1983.

2. Trip SA, Schlottmann PG, Jones SJ, Altmann DR, Garway-Heath DF, Thompson AJ, Plant GT and Miller DH: Retinal nerve fiber layer axonal loss and visual dysfunction in optic neuritis. Ann Neurol 58: 383-391, 2005.

3. Fisher JB, Jacobs DA, Markowitz CE, Galetta SL, Volpe NJ, Nano-Schiavi ML, Baier ML, Frohman EM, Winslow H and Frohman TC: Relation of visual function to retinal nerve fiber layer thickness in multiple sclerosis. Ophthalmology 113: 324-332, 2006.

4. Costello F, Coupland S, Hodge W, Lorello GR, Koroluk J, Pan YI, Freedman MS, Zackon DH and Kardon RH: Quantifying axonal loss after optic neuritis with optical coherence tomography. Ann Neurol 59: 963-969, 2006.

5. Parisi V, Manni G, Spadaro M, Colacino G, Restuccia R, Marchi S, Bucci MG and Pierelli F: Correlation between morphological and functional retinal impairment in multiple sclerosis patients. Invest Ophthalmol Vis Sci 40: 2520-2527, 1999.

6. Henderson AP, Trip SA, Schlottmann PG, Altmann DR, Garway-Heath DF, Plant GT and Miller DH: An investigation of the retinal nerve fibre layer in progressive multiple sclerosis using optical coherence tomography. Brain 131: 277-287, 2008. 
7. Pulicken M, Gordon-Lipkin E, Balcer LJ, Frohman E, Cutter G and Calabresi PA: Optical coherence tomography and disease subtype in multiple sclerosis. Neurology 69: 2085-2092, 2007.

8. Ikuta $\mathrm{F}$ and Zimmerman HM: Distribution of plaques in seventy autopsy cases of multiple sclerosis in the United States. Neurology 26: 26-28, 1976.

9. Toussaint D, Périer O, Verstappen A and Bervoets S: Clinicopathological study of the visual pathways, eyes, and cerebral hemispheres in 32 cases of disseminated sclerosis. J Clin Neuroophthaloml 3: 211-220, 1983.

10. Green AJ, McQuaid S, Hauser SL, Allen IV and Lyness R: Ocular pathology in multiple sclerosis: Retinal atrophy and inflammation irrespective of disease duration. Brain 133: 1591-1601, 2010.

11. Jindahra P, Petrie A and Plant GT: Retrograde trans-synaptic retinal ganglion cell loss identified by optical coherence tomography. Brain 132: 628-634, 2009.

12. Bridge H, Jindahra P, Barbur J and Plant GT: Imaging reveals optic tract degeneration in hemianopia. Invest Ophthalmol Vis Sci 52 382-388, 2011.

13. Cowey A, Alexander I and Stoerig P: Transneuronal retrograde degeneration of retinal ganglion cells and optic tract in hemianopic monkeys and humans. Brain 134: 2149-2157, 2011.

14. Gabilondo I, Martínez-Lapiscina EH, Martínez-Heras E, Fraga-Pumar E, Llufriu S, Ortiz S, Bullich S, Sepulveda M, Falcon $\mathrm{C}$, Berenguer J, et al: Trans-synaptic axonal degeneration in the visual pathway in multiple sclerosis. Ann Neurol 75: 98-107, 2014

15. Pfueller CF, Brandt AU, Schubert F, Bock M, Walaszek B, Waiczies H, Schwenteck T, Dörr J, Bellmann-Strobl J, Mohr C, et al: Metabolic changes in the visual cortex are linked to retinal nerve fiber layer thinning in multiple sclerosis. PLoS One 6: e18019, 2011.

16. Sinnecker T, Oberwahrenbrock T, Metz I, Zimmermann H, Pfueller CF, Harms L, Ruprecht K, Ramien C, Hahn K, Brück W, et al: Optic radiation damage in multiple sclerosis is associated with visual dysfunction and retinal thinning - an ultrahigh-field MR pilot study. Eur Radiol 25: 122-131, 2015

17. Sepulcre J, Masdeu JC, Pastor MA, Goñi J, Barbosa C, Bejarano B and Villoslada P: Brain pathways of verbal working memory: A lesion-function correlation study. Neuroimage 47: 773-778, 2009.

18. Audoin B, Fernando KT, Swanton JK, Thompson AJ, Plant GT and Miller DH: Selective magnetization transfer ratio decrease in the visual cortex following optic neuritis. Brain 129: 1031-1039, 2006

19. Sepulcre J, Murie-Fernandez M, Salinas-Alaman A, García-Layana A, Bejarano B and Villoslada P: Diagnostic accuracy of retinal abnormalities in predicting disease activity in MS. Neurology 68: 1488-1494, 2007.

20. Toledo J, Sepulcre J, Salinas-Alaman A, García-Layana A, Murie-Fernandez M, Bejarano B and Villoslada P: Retinal nerve fiber layer atrophy is associated with physical and cognitive disability in multiple sclerosis. Mult Scler 14: 906-912, 2008.

21. Gordon-Lipkin E, Chodkowski B, Reich DS, Smith SA, Pulicken M, Balcer LJ, Frohman EM, Cutter G and Calabresi PA: Retinal nerve fiber layer is associated with brain atrophy in multiple sclerosis. Neurology 69: 1603-1609, 2007.

22. Grazioli E,Zivadinov R, Weinstock-Guttman B, Lincoff N, Baier M Wong JR, Hussein S, Cox JL, Hojnacki D and Ramanathan M: Retinal nerve fiber layer thickness is associated with brain MRI outcomes in multiple sclerosis. J Neurol Sci 268: 12-17, 2008.

23. Siger M, Dziegielewski K, Jasek L, Bieniek M, Nicpan A, Nawrocki J and Selmaj K: Optical coherence tomography in multiple sclerosis: Thickness of the retinal nerve fiber layer as a potential measure of axonal loss and brain atrophy. J Neurol 255: 1555-1560, 2008

24. Saidha S, Sotirchos ES, Oh J, Syc SB, Seigo MA, Shiee N, Eckstein C, Durbin MK, Oakley JD, Meyer SA, et al: Relationships between retinal axonal and neuronal measures and global central nervous system pathology in multiple sclerosis. JAMA Neurol 70: 34-43, 2013.

25. Dörr J, Wernecke KD, Bock M, Gaede G, Wuerfel JT, Pfueller CF, Bellmann-Strobl J, Freing A, Brandt AU and Friedemann P: Association of retinal and macular damage with brain atrophy in multiple sclerosis. PLoS One 6: e18132, 2011.

26. Zimmermann H, Freing A, Kaufhold F, Gaede G, Bohn E, Bock M, Oberwahrenbrock T, Young KL, Dörr J, Wuerfel JT, et al: Optic neuritis interferes with optical coherence tomography and magnetic resonance imaging correlations. Mult Scler 19: 443-450, 2013

27. The IFN $\beta$ Multiple Sclerosis Study Group: Interferon beta-1b is effective in relapsing-remitting multiple sclerosis. I. Clinical results of a multicenter, randomized, double-blind, placebo-controlled trial. Neurology 43: 655-661, 1993.
28. Jacobs LD, Cookfair DL, Rudick RA, Herndon RM, Richert JR, Salazar AM, Fischer JS, Goodkin DE, Granger CV, Simon JH, et al; The Multiple Sclerosis Collaborative Research Group (MSCRG): Intramuscular interferon beta-1a for disease progression in relapsing multiple sclerosis. Ann Neurol 39: 285-294, 1996

29. Ebers GC; PRISMS Study Group: Randomised double-blind placebo-controlled study of interferon beta-1a in relapsing/remitting multiple sclerosis. PRISMS (Prevention of Relapses and Disability by Interferon beta-1a Subcutaneously in Multiple Sclerosis) Study Group. Lancet 352: 1498-1504, 1998.

30. Goodin DS, Traboulsee A, Knappertz V, Reder AT, Li D, Langdon D, Wolf C, Beckmann K, Konieczny A and Ebers GC; 16-Year Long Term Follow-up Study Investigators: Relationship between early clinical characteristics and long term disability outcomes: 16 year cohort study (follow-up) of the pivotal interferon $\beta-1 \mathrm{~b}$ trial in multiple sclerosis. J Neurol Neurosurg Psychiatry 83: 282-287, 2012.

31. Ebers GC, Traboulsee A, Li D, Langdon D, Reder AT, Goodin DS, Bogumil T, Beckmann K, Wolf C and Konieczny A; Investigators of the 16-year Long-Term Follow-Up Study: Analysis of clinical outcomes according to original treatment groups 16 years after the pivotal IFNB-1b trial. J Neurol Neurosurg Psychiatry 81: 907-912, 2010.

32. Sühs KW, Hein K, Pehlke JR, Käsmann-Kellner B and Diem R: Retinal nerve fibre layer thinning in patients with clinically isolated optic neuritis and early treatment with interferon-beta. PLoS One 7: e51645, 2012.

33. Tugcu B, Soysal A, Kilic M, Yuksel B, Kale N, Yigit U and Arpaci B: Assessment of structural and functional visual outcomes in relapsing remitting multiple sclerosis with visual evoked potentials and optical coherence tomography. J Neurol Sci 335: 182-185, 2013

34. Polman CH, Reingold SC, Edan G, Filippi M, Hartung HP, Kappos L, Lublin FD, Metz LM, McFarland HF, O'Connor PW, et al: Diagnostic criteria for multiple sclerosis: 2005 revisions to the 'McDonald Criteria'. Ann Neurol 58: 840-846, 2005.

35. Schippling S, Balk LJ, Costello F, Albrecht P, Balcer L, Calabresi PA, Frederiksen JL, Frohman E, Green AJ, Klistorner A, et al: Quality control for retinal OCT in multiple sclerosis: Validation of the OSCAR-IB criteria. Mult Scler 21: 163-170, 2015

36. Tewarie P, Balk L, Costello F, Green A, Martin R, Schippling S and Petzold A: The OSCAR-IB consensus criteria for retinal OCT quality assessment. PLoS One 7: e34823, 2012.

37. Fan Q, Teo YY and Saw SM: Application of advanced statistics in ophthalmology. Invest Ophthalmol Vis Sci 52: 6059-6065, 2011.

38. Zeger SL and Liang KY: Longitudinal data analysis for discrete and continuous outcomes. Biometrics 42: 121-130, 1986.

39. Mancl LA and DeRouen TA: A covariance estimator for GEE with improved small-sample properties. Biometrics 57: 126-134, 2001

40. Højsgaard S, Halekoh U and Yan J: The R Package geepack for Generalized Estimating Equations. J Stat Softw 15: 1-11, 2006.

41. Araie M: Test-retest variability in structural parameters measured with glaucoma imaging devices. Jpn J Ophthalmol 57: 1-24, 2013.

42. Talman LS, Bisker ER, Sackel DJ, Long DA Jr, Galetta KM, Ratchford JN, Lile DJ, Farrell SK, Loguidice MJ, Remington $\mathrm{G}$, et al: Longitudinal study of vision and retinal nerve fiber layer thickness in multiple sclerosis. Ann Neurol 67: 749-760, 2010.

43. García-Martín E, Pueyo V, Fernández J, Almárcegui C, Dolz I, Martín J, Ara JR and Honrubia FM: Atrophy of the retinal nerve fibre layer in multiple sclerosis patients. Prospective study with two years follow-up. Arch Sociedad Esp Oftalmol 85: 179-186, 2010 (In Spanish).

44. Galetta KM, Graves J, Talman LS, Lile DJ, Frohman EM, Calabresi PA, Galetta SL and Balcer LJ: Visual pathway axonal loss in benign multiple sclerosis: A longitudinal study. J Neuroophthalmol 32: 116-123, 2012.

45. Kimbrough DJ, Sotirchos ES, Wilson JA, Al-Louzi O, Conger A, Conger D, Frohman TC, Saidha S, Green AJ, Frohman EM, et al: Retinal damage and vision loss in African American multiple sclerosis patients. Ann Neurol 77: 228-236, 2015. 
46. Waubant E, Maghzi AH, Revirajan N, Spain R, Julian L, Mowry EM, Marcus J, Liu S, Jin C, Green A, et al: A randomized controlled phase II trial of riluzole in early multiple sclerosis. Ann Clin Transl Neurol 1: 340-347, 2014.

47. Henderson AP, Trip SA, Schlottmann PG, Altmann DR, Garway-Heath DF, Plant GT and Miller DH: A preliminary longitudinal study of the retinal nerve fiber layer in progressive multiple sclerosis. J Neurol 257: 1083-1091, 2010.

48. Serbecic N, Aboul-Enein F, Beutelspacher SC, Vass C, Kristoferitsch W, Lassmann H, Reitner A and Schmidt-ErfurthU: High resolution spectral domain optical coherence tomography (SD-OCT) in multiple sclerosis: The first follow up study over two years. PLoS One 6: e19843, 2011.

49. Petzold A, de Boer JF, Schippling S, Vermersch P, Kardon R, Green A, Calabresi PA and Polman C: Optical coherence tomography in multiple sclerosis: A systematic review and meta-analysis. Lancet Neurol 9: 921-932, 2010.

50. Sobaci G, Demirkaya S, Gundogan FC and Mutlu FM: Stereoacuity testing discloses abnormalities in multiple sclerosis without optic neuritis. J Neuroophthalmol 29: 197-202, 2009.

51. Villoslada P, Cuneo A, Gelfand J, Hauser SL and Green A Color vision is strongly associated with retinal thinning in multiple sclerosis. Mult Scler 18: 991-999, 2012.

52. Almarcegui C, Dolz I, Pueyo V, Garcia E, Fernandez FJ, Martin J, Ara JR and Honrubia F: Correlation between functional and structural assessments of the optic nerve and retina in multiple sclerosis patients. Clin Neurophysiol 40: 129-135, 2010.

53. Davis AS, Hertza J, Williams RN, Gupta AS and Ohly JG: The influence of corrected visual acuity on visual attention and incidental learning in patients with multiple sclerosis. Appl Neuropsychol 16: 165-168, 2009.

54. Bruce JM, Bruce AS and Arnett PA: Mild visual acuity disturbances are associated with performance on tests of complex visual attention in MS. J Int Neuropsychol Soc 13: 544-548, 2007.
55. Wieder L, Gäde G, Pech LM, Zimmermann H, Wernecke KD, Dörr JM, Bellmann-Strobl J, Paul F and Brandt AU: Low contrast visual acuity testing is associated with cognitive performance in multiple sclerosis: A cross-sectional pilot study. BMC Neurol 13: 167,2013

56. Boutros T, Croze E and Yong VW: Interferon-beta is a potent promoter of nerve growth factor production by astrocytes. J Neurochem 69: 939-946, 1997.

57. Biernacki K, Antel JP, Blain M, Narayanan S, Arnold DL and Prat A: Interferon beta promotes nerve growth factor secretion early in the course of multiple sclerosis. Arch Neurol 62: 563-568, 2005.

58. Jin S, Kawanokuchi J, Mizuno T, Wang J, Sonobe Y, Takeuchi H and Suzumura A: Interferon-beta is neuroprotective against the toxicity induced by activated microglia. Brain Res 1179: 140-146, 2007.

59. Croze E, Yamaguchi KD, Knappertz V, Reder AT and Salamon $\mathrm{H}$ : Interferon-beta-1b-induced short- and long-term signatures of treatment activity in multiple sclerosis. Pharmacogenomics J 13: 443-451, 2013.

60. Zivadinov R, Reder AT, Filippi M, Minagar A, Stüve O, Lassmann H, Racke MK, Dwyer MG, Frohman EM and Khan O: Mechanisms of action of disease-modifying agents and brain volume changes in multiple sclerosis. Neurology 71: 136-144, 2008.

61. Filippi M, Rocca MA, Camesasca F, Cook S, O'Connor P, Arnason BG, Kappos L, Goodin D, Jeffery D, Hartung HP, et al: Interferon $\beta-1 \mathrm{~b}$ and glatiramer acetate effects on permanent black hole evolution. Neurology 76: 1222-1228, 2011.

62. Sahraian MA, Radue EW, Haller S and Kappos L: Black holes in multiple sclerosis: Definition, evolution, and clinical correlations. Acta Neurol Scand 122: 1-8, 2010.

63. Bock M, Brandt AU, Dörr J, Pfueller CF, Ohlraun S, Zipp F and Paul F: Time domain and spectral domain optical coherence tomography in multiple sclerosis: A comparative cross-sectional study. Mult Scler 16: 893-896, 2010. 